Mathematical Modelling and Analysis

Volume 6 Number 2, 2001, pages 300-309

(C) 2001 Technika

\title{
PHASE TRANSITION AND HYSTERESIS ANALYSIS FOR SHAPE MEMORY ALLOYS APPLICATIONS
}

\author{
R.V.N. MELNIK \\ University of Southern Denmark \\ MCI, Grundtvigs Alle 150, Sonderborg, DK-6400, Denmark \\ E-mail: rmelnik@mci.sdu.dk
}

Received September 28, 2001

\begin{abstract}
Center manifold approximations for the 1D Landau-Devonshire and 3D Falk-Konopka models are applied to modelling phase transitions in shape memory alloys. The methodology, allowing a systematic improvement of mathematical models for phase transitions and hysteresis phenomena, is explained, and typical computational results with such low-dimensional models are presented.
\end{abstract}

\section{INTRODUCTION: PHASE-FIELDS MODELS, COUPLING PHENOMENA, AND HYSTERESIS}

Practical materials science has always provided mathematicians with challenging and important problems which stimulated the development of new numerical methods, new mathematical modelling tools, and generated new ideas applicable in other fields of engineering applications. Indeed, the basic physics of complicated materials is still only partially known, and when it comes to dynamic non-equilibrium phenomena in such materials the complexity of associated problems increases substantially.

My major focus in this paper is on materials with memory, in particular on shape memory alloys (SMA). The shape memory effect was discovered around 1938 by A. Greninger, V.G. Mooradian, and G.V. Kurdumov, but only recently the use of shape-memory materials has become a viable technology with an industry growing at more than one quarter of its size each year. I aim at a systematic description of coupled thermomechanical fields in such 
materials and my basic tools here will be center manifold theory $[4 ; 19 ; 20]$ and numerical techniques for solving differential-algebraic equations.

The approach to modelling in materials science adopted in this paper goes back to works by Landau and Khalatnikov who assumed that a continuous, possibly multicomponent, conserved or nonconserved field function (order parameter or phase-field parameter) can be used to characterized the phase of a material. Then, the state of the material can be described by the temperature, order parameter, and, perhaps, some other state variables, so that by using principles of irreversible thermodynamics one can proceed to the model construction by deriving evolution equations for this state. Such models are known as phase-field models and their mathematical analysis has been a subject of a number of recent papers (e.g., [10] and references therein). Examples of phase-field models where this idea is used include the Allen-Cahn model for the description of polycrystalline solids, the Penrose-Fife model (of which the Cahn-Hilliard model is a special case), and many others. The phenomena described by such phase-field models are quite complex and often require incorporating hysteresis coming naturally from nonlinear dynamics, coupling (e.g., via constitutive relations), and/or consideration of nonconvex potentials. Although hysteresis comes naturally into consideration in such classical fields as plasticity, ferromagnetism, ferroelectricity, its rigorous treatment has been initiated relatively recently with works by Krasnoselskii \& Pokrovskii (see [10] and references therein). This phenomenon has to be dealt with in shape memory applications as well, in particular in the pseudoelastic range. In fact, at the macroscopic level three main patterns of behaviour of shape memory alloys are of interest [2]: (a) the pseudoelastic effect (large elastic deformations resulted from loading can be recovered through hysteresis upon unloading); (b) the shape memory effect (large residual deformations resulted from loading-unloading can be recovered upon heating); (c) thermal hysteresis effect (the ability to reproduce both deformed (low temperature) and undeformed (high temperature) states upon cooling/heating at constant load). Each of these patterns can be clearly observed with the models discussed here (see $[13 ; 14]$ and references therein for further details). What is even more important, these models can be systematically improved to capture new features of the dynamics by using the methodology applied in this paper.

\section{THE LANDAU CRITERION AND GOVERNING EQUA- TIONS FOR SMA PHASE TRANSITIONS}

Several different methodologies have been proposed in the literature in order to adequately describe the dynamics of phase transitions between different equilibrium configurations of the metallic lattice of SMA, known as austenite (high temperature phase), and martensite (low temperature phase, and, ultimately, to control this dynamics. Earlier mathematical models developed in this field had a number of limitations [21] related to the treatment of spatial effects and thermomechanical coupling, and at a large extent those limitations 
are inherited by some of multi-well mixture approaches [7]. In attempts to improve those earlier models several groups of models have been developed in the literature, main of them are based on phenomenological constitutive laws with or without volume fraction as internal variables, phase diagram kinetics, and so-called micro-macro thermomechanical models (e.g., $[2 ; 7 ; 11$; 16]). In this paper, we base our consideration on the Landau-Devonshiretype models and their generalization to the 3D case in the spirit of Falk \& Konopka (see $[13 ; 14]$ and references therein). However, the same idea can be applied to other models in this field and our main goal in this paper is to explain foundations and to show the effectiveness of center manifold technique in capturing main features of the dynamics of phase transitions, allowing a systematic improvement of the resulting computational models.

The basis for modelling is the Landau criterion stating that any isothermal equilibrium configuration of the lattice corresponds to a minimum (global or local) of the free energy function. The free energy function strongly depends upon the statistics of the phenomenon and has to be derived from a statistical model (see also [6] for other non-trivial issues related to the choice of the free energy function). Our consideration in this paper is pertinent to the following general form of this function:

$$
\Psi(\boldsymbol{\epsilon}, \theta)=\psi^{0}(\theta)+\sum_{i=1}^{\infty} \psi^{i}(\boldsymbol{\epsilon}, \theta), \quad \psi^{n}=\sum_{j=1}^{j^{n}} \psi_{j}^{n} \mathcal{I}_{j}^{n}, \quad \psi^{0}(\theta)=\psi_{0}^{0}(\theta)
$$

where $\epsilon$ is the deformation gradient, $\theta$ is the temperature of the system $(\theta>0$, $\left.\inf _{(\boldsymbol{x}, t)} \theta=0\right), \psi^{n}, n=1,2, \ldots$ are strain invariants that define independent material parameters of the $n$-th order, and $\mathcal{I}_{j}^{n}$ are the corresponding strain invariants. The upper limit of the sum in $(2.1), j^{n}$, is chosen as the number of all invariant directions associated with a representation of the 48th order cubic symmetry group of the parent (austenite) phase in order to make the free energy function invariant with respect to the symmetry group of austenite.

The equation of motion, written with respect to displacements $\boldsymbol{u}$, has the following form

$$
\rho \frac{\partial^{2} \boldsymbol{u}}{\partial t^{2}}=\nabla \cdot \boldsymbol{s}+\boldsymbol{F} \quad \text { with } \quad \boldsymbol{F}=\rho(\boldsymbol{f}+\hat{\boldsymbol{f}})-\hat{\rho} \boldsymbol{v}
$$

where $\rho$ is the density of the material, $f$ is a given body force per unit mass, $\hat{\rho}$ and $\hat{f}$ are nonlocal mass and force residuals respectively, $\boldsymbol{v}=\partial \boldsymbol{u} / \partial t$ is the velocity vector, and $s$ is the stress tensor. The energy balance equation can be represented in the form

$$
\rho \frac{\partial e}{\partial t}-\boldsymbol{s}^{T}:(\nabla \boldsymbol{v})+\nabla \cdot \boldsymbol{q}=g
$$

where $e$ is the internal energy (per unit mass) of the system, $\boldsymbol{q}$ is the heat 
flux, $\boldsymbol{a}^{T}: \boldsymbol{b}=\sum_{i, j=1}^{3} a_{i j} b_{i j}$ is the standard notation for the rank 2 tensors $\boldsymbol{a}$ and $\boldsymbol{b}$, and $g=\rho(h+\hat{h})-\rho \hat{\boldsymbol{f}} \cdot \boldsymbol{v}-\hat{\rho}\left(e-\frac{\boldsymbol{v}^{2}}{2}\right)$ with $h$ being the heat source density, and $\hat{h}$ is the nonlocal energy residual (see [13] and references therein for conditions on localized residuals). The right-hand sides of equations (2.2) and (2.3) incorporate into the model nonlocal and dissipative effects of thermomechanical waves.

We assume that there exists a one-to-one entropy function of the system state and denote its density by $\eta$. Thermodynamic potential, invariant under a time shift, is defined in the form of the Helmholtz free energy through $\eta$, internal energy, and the temperature of the system as $\Psi=e-\theta \eta$ with $\eta=-\frac{\partial \Psi}{\partial \theta}$. Having the free energy function, we define the shear stress by its three components: the quasi-conservative component, $s^{q}$, the stress component due to mechanical dissipation, $s^{m}$, and the stress component due to thermal dissipations, $s^{t}$, (the latter is assumed to be negligible).

The specific form of the free energy function is chosen in such a way that its minima correspond to different phases of the material, and depending on the value of temperature, we mimic the behaviour of the material which can alternate between a single thermodynamically unstable nonmonotone branch and multiple unstable branches. The coupling between stresses, deformation gradients, temperature and heat fluxes is specified by constitutive relations

$$
\Phi_{1}(\boldsymbol{s}, \boldsymbol{\epsilon})=0, \quad \Phi_{2}(\boldsymbol{q}, \theta)=0,
$$

where it is implicitly assumed that these relations may involve spatial and temporal derivatives of the functions. The specification of $\Phi_{2}$ is made by using the principles of extended thermodynamics, requiring the hyperbolic Cattaneo-Vernotte model to be satisfied

$$
\boldsymbol{q}+\tau_{0} \frac{\partial \boldsymbol{q}}{\partial t}=-k(\theta, \boldsymbol{\epsilon}) \nabla \theta
$$

where $\tau_{0}$ is the dimensionless thermal relaxation time and $k(\theta, \boldsymbol{\epsilon})$ is the thermal conductivity of the material (typically $k=1+\tilde{\beta} \theta$ with the given dimensionless coefficient $\tilde{\beta}$ ).

To specify functional dependency $\Phi_{1}$ we start from the 1D case of the Landau-Devonshire form for the free energy function (e.g., [8; 15; 21]):

$$
\Psi(\theta, \epsilon)=\psi_{0}(\theta)+\psi_{1}(\theta) \psi_{2}(\epsilon)+\psi_{3}(\epsilon),
$$

where $\psi_{0}(\theta)=\alpha_{0}-\alpha_{1} \theta \ln \theta$ models thermal field contributions, $\psi_{1}(\theta) \psi_{2}(\epsilon)$ (with $\psi_{1}(\theta)=\frac{1}{2} \alpha_{2} \theta$ and $\psi_{2}(\epsilon)=\epsilon^{2}$ ) models shape-memory contributions, and $\psi_{3}(\epsilon)=-\frac{1}{2} \alpha_{2} \theta_{1} \epsilon^{2}-\frac{1}{4} \alpha_{4} \epsilon^{4}+\frac{1}{6} \alpha_{6} \epsilon^{6}$ models mechanical field contributions 
(all $\alpha_{i}$ and $\theta_{1}$ are positive constants). Such a choice of the Helmholtz free energy function satisfies all requirements of the Landau theory of structural phase transitions, or more precisely, its modification to account for 6th order polynomials (a polynomial of the 4th order cannot describe twinning effects). Note that, as it is pointed out in [13], with reported values of $\gamma \sim 10^{-10}$ $10^{-12}$, accounting for the Ginzburg term $\left(\gamma / 2 \epsilon_{x}^{2}\right)$ has a negligible effect in the series of computational experiments conducted in this paper and in [13; 14]. Then, the functional dependency of $\Phi_{1}$ is taken as

$$
s=\rho\left[p(\theta, \epsilon)+\lambda\left(\frac{\partial \theta}{\partial t}, \frac{\partial \epsilon}{\partial t}\right)\right]
$$

where $p(\theta, \epsilon)=\frac{\partial \Psi}{\partial \epsilon}$ and $\lambda\left(\frac{\partial \theta}{\partial t}, \frac{\partial \epsilon}{\partial t}\right)=\tilde{\mu}(\theta) \frac{\partial \epsilon}{\partial t}+\tilde{\nu}(\epsilon) \frac{\partial \theta}{\partial t}$ with $\tilde{\mu}$ and $\tilde{\nu}$ being material-specific coefficients that characterize the dependency of the stress on the rate of the deformation gradient and temperature, respectively.

In the 3D case the situation is much more involved. Using (2.1) and FalkKonopka assumptions valid for the copper-based alloys (see [14] and references therein) it is possible to reduce the number of required parameters in the $3 \mathrm{D}$ case from 32 to only 10 material constants (or more generally, temperature dependent functions)

$$
\Psi=\psi^{0}(\theta)+\sum_{j=1}^{3} \psi_{j}^{2} \mathcal{I}_{j}^{2}+\sum_{j=1}^{5} \psi_{j}^{4} \mathcal{I}_{j}^{4}+\sum_{j=1}^{2} \psi_{j}^{6} \mathcal{I}_{j}^{6},
$$

where, for example, the $\mathrm{Cu}_{14} \mathrm{Al}_{3} \mathrm{Ni}_{83}$ strain invariant $\mathcal{I}_{2}^{2}$ is determined by

$$
\mathcal{I}_{2}^{2}=\frac{1}{12}\left(2 \epsilon_{33}-\epsilon_{11}-\epsilon_{22}\right)^{2}+\frac{1}{4}\left(\epsilon_{11}-\epsilon_{22}\right)^{2} .
$$

The corresponding (to $\mathcal{I}_{2}^{2}$ ) material coefficient is temperature dependent

$$
\psi_{2}^{2}=\left(1.41 \times 10^{5}+46(\theta-300)\right) \mathrm{g} /\left(\mathrm{ms}^{2} \mathrm{~cm}\right) .
$$

\section{CENTER MANIFOLD TECHNIQUE IN MODELLING SHAPE-MEMORY-ALLOY DYNAMICS}

Several computational approaches have been already applied to modelling dynamics of phase transitions and hysteresis phenomena in shape memory alloy materials (e.g., $[7 ; 8 ; 15]$ and references therein). However, as was pointed out in [7], the phenomenon of phase transitions in SMA materials is "sufficiently complex such that any computational treatment attempting to capture the macroscopic response of a structural system composed of these materials necessarily requires significant simplifications to result in a tractable problem". 
Such systematic simplifications of models describing the dynamics of SMA are proposed here via the application of center manifold theory.

Reduction procedures of original models on center manifolds are used widely, for example, in different approaches for computing normal forms applied to the analysis of dynamical systems described by differential equations. Normal forms are quite useful in the analysis of non-linear oscillations [9], and the normal form methodology and center manifold theory are often considered together, because a sequence of successive non-linear transformations is actually reduces the original system to a center manifold associated with the critical modes. The current methodology of the normal form method is attributed to Bruno (e.g., [3] and references therein) who published his first works on the topic in the early 1960-ies. Approximately at the same time the first works on center manifold theory have been published by Pliss (see $[5 ; 17 ; 18]$ for the latest theoretical development of this theory and references therein to earlier works). After the appearance of a classical monograph by James Carr [4] the interest in an effective combination of these two intrinsically connected methodologies has been growing [5].

Invariant sets are at the heart of studying dynamics [17]. Center manifold theory is a method of reducing the system dimensionality and restricting attention to an invariant subspaces (the center manifold) which contains all of the essential dynamic behaviour of the system. The fundamental potential in the applicability of center manifold theory to such complex problems as the dynamics of phase transitions is that the methodology can deal successfully with such cases where invariant sets (or invariant manifolds of the dynamics described by differential equations) might become non-hyperbolic. Since such sets cannot persist in general [12] the problem is to construct a normally hyperbolic (invariant) manifold which contains the invariant set of the smallest possible dimension. This manifold is known as a center manifold. The construction of such a manifold is a challenging and, generally speaking, tremendous task. With the advance of computer algebra this construction can be performed effectively. This idea has been applied for the first time to shape-memory alloys models by the author and his collaborators $[13 ; 14]$.

The basic idea of our approach is to express the physical fields in terms of asymptotic sums in some vibrational amplitudes and their gradients, and then, to find an asymptotic approximation to the system of PDEs describing the dynamics of SMA by using computer algebra. Such a low dimensional model is derived systematically, up to the arbitrary order of accuracy. In fact, it is this link of center manifold theory with computer algebra that makes this tool one of the most powerful tools in the analysis of complex mathematical models.

To demonstrate the basic principles of the methodology, we follow [14] and consider a shape memory alloy slab with large extend in the $x=x_{1}$ direction compared to its thickness of $2 b$ in the $y=x_{2}$ direction $(-b<y<b)$ and neglect any motion and dependence in the $x_{3}$ direction. In this case all related physical fields can be expressed in terms of the amplitudes of the cross- 
sectional averages of critical modes, remaining the leading order structure of the critical eigenmodes constant across the slab [14]:

$$
U_{i}(x, t)=\overline{u_{i}}, \quad V_{i}(x, t)=\overline{v_{i}}, \quad \Theta^{\prime}(x, t)=\overline{\theta^{\prime}},
$$

where an over bar denotes the $y$-average quantity and $\theta^{\prime}=\theta-\theta_{0}\left(\theta_{0}=\right.$ $300^{\circ} \mathrm{K}$ ). Then, we seek a model that is written in terms of amplitudes (3.1). In other words, we seek an invariant (low-dimensional) manifold upon which these amplitudes evolve "slowly|:

$$
u_{i}=\mathcal{U}_{i}\left(\boldsymbol{U}, \boldsymbol{V}, \Theta^{\prime}\right), \quad v_{i}=\mathcal{V}_{i}\left(\boldsymbol{U}, \boldsymbol{V}, \Theta^{\prime}\right), \quad \theta=\mathcal{T}\left(\boldsymbol{U}, \boldsymbol{V}, \Theta^{\prime}\right), \quad i=1,2,
$$

where

$$
\frac{\partial U_{i}}{\partial t}=V_{i}, \quad \frac{\partial V_{i}}{\partial t}=g_{i}\left(\boldsymbol{U}, \boldsymbol{V}, \Theta^{\prime}\right), \quad \frac{\partial \Theta^{\prime}}{\partial t}=g_{\theta}\left(\boldsymbol{U}, \boldsymbol{V}, \Theta^{\prime}\right) .
$$

The last set of relationships is the reason why center manifold theory is often regarded as a certain generalization of ideas coming from the implicit theorem to the analysis of ordinary and partial differential equations. Now, we have to substitute (3.2) into the original model $(2.2),(2.3)$ and to solve the resulting system with respect to small parameters $\partial_{x}, E=\left\|\boldsymbol{U}_{x}\right\|+\left\|\boldsymbol{V}_{x}\right\|$, and $\vartheta=\left\|\Theta^{\prime}\right\|$ (see details in [14]). This results in approximations of (3.2), which can be obtained up to the arbitrary order accuracy. For this purpose we use the computer algebra package REDUCE. Specific approximations obtained along this avenue of ideas can be found in [13; 14]. These approximations are used after the solution of the corresponding system with respect to $\boldsymbol{U}, \boldsymbol{V}$, and $\Theta$. The system for the center manifold approximation of the 3D Falk-Konopka model has been derived in [14] and in the case when the slab is thin enough so that in effect $b=0$ this system can be written in the form differential-algebraic equations as follows

$$
\left\{\begin{array}{l}
\rho \frac{\partial V_{1}}{\partial t}=\frac{\partial s}{\partial x}+F, \quad \frac{\partial U_{1}}{\partial t}=V_{1} \\
C_{v} \frac{\partial \Theta^{\prime}}{\partial t}=k \frac{\partial^{2} \Theta^{\prime}}{\partial x^{2}}+\left(c_{11}+c_{12} \Theta^{\prime}-c_{13}\left(\Theta^{\prime}\right)^{2}\right) \frac{\partial U_{1}}{\partial x} \frac{\partial V_{1}}{\partial x}+ \\
\quad+\left(c_{14}+c_{15} \theta^{\prime}\right) \frac{\partial V_{1}}{\partial x}\left(\frac{\partial U_{1}}{\partial x}\right)^{3}+c_{18} \frac{\partial V_{1}}{\partial x}\left(\frac{\partial U_{1}}{\partial x}\right)^{5}+g \\
s=\left(c_{1}+c_{2} \theta^{\prime}-c_{3}\left(\theta^{\prime}\right)^{2}\right) \frac{\partial U_{1}}{\partial x}-\left(c_{4}-c_{5} \theta^{\prime}\right)\left(\frac{\partial U_{1}}{\partial x}\right)^{3}+c_{6}\left(\frac{\partial U_{1}}{\partial x}\right)^{5}
\end{array}\right.
$$

where all coefficients $c_{k}$ are positive material constants. It should be emphasized that the vector field of the original dynamics in the construction of the center manifold will typically changed, and therefore an important issue to 
address is the appropriate approximations of boundary and initial conditions for the center manifold approximation of the dynamics. These issues are outside the scope of the present paper, but the interested reader can consult [19; $20]$ and references therein for further details.

Center manifold models for the classical 1D Landau-Devonshire model and the 3D dimensional Falk-Konopka model, as described in Section 2, have been used to model phase transitions and hysteresis phenomena in shape memory alloys. Time dependent external actions on the system (stresses and heat fluxes at the boundary, as well as distributed over the domain) are easily treated with the derived models. In particular, experiments have been carried out for thermal control of phase transitions with constant mechanical forcing and time-varying thermal forcing chosen as $A \pi \sin ^{3}(t \pi / B)$ (in $\mathrm{g} /\left(\mathrm{ms}^{3} \mathrm{~cm}\right)$ units) with various coefficients $A$ and $B$. A typical result from this series of experiments is given in Fig. 1, where martensitic-austenitic transitions are clearly reproduced.
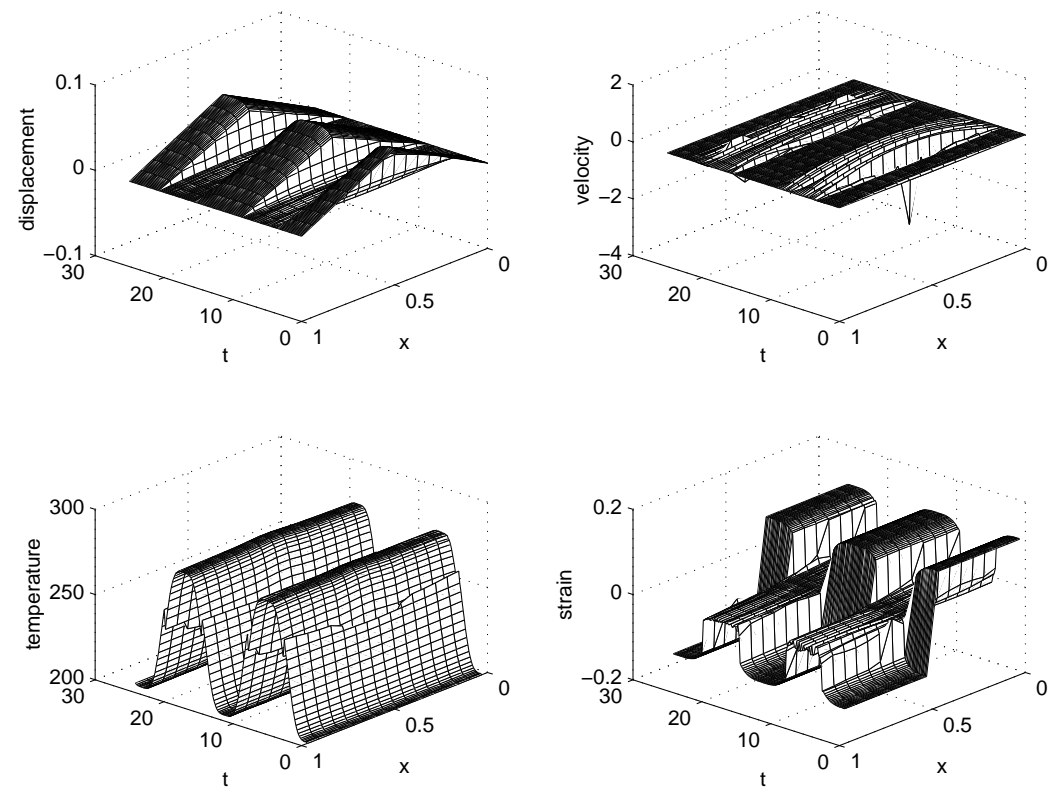

Figure 1. Typical results from the shape memory effect modelling: thermally induced phase transitions.

A number of experiments related to mechanical control of phase transitions (without thermal forcing, but with time-varying mechanical forcing) has also been performed. It has been also demonstrated that boundary stress control of phase transitions might not be sufficient to induce martensitic-austenitic transitions, while a combined effect of boundary stress and distributed temperature can produce the desired result (see Fig. 2 and further details in [13]). Details of computational experiments on "ferroelastic"- and "pseudoelastic"- 
like hysteresis can be found in [13], where the behaviour of temperature-strain relations has also been analyzed and the "parabolic"-like shape of temperaturestrain hysteresis loops obtained with Landau-Devonshire types models have been explained for the first time.

The same methodology, as described here, can be applied to models other than Landau-Devonshire or Falk-Konopka models, providing an efficient tool for a systematic improvement of mathematical models for phase transitions and hysteresis phenomena, and making computations tractable for engineering applications.
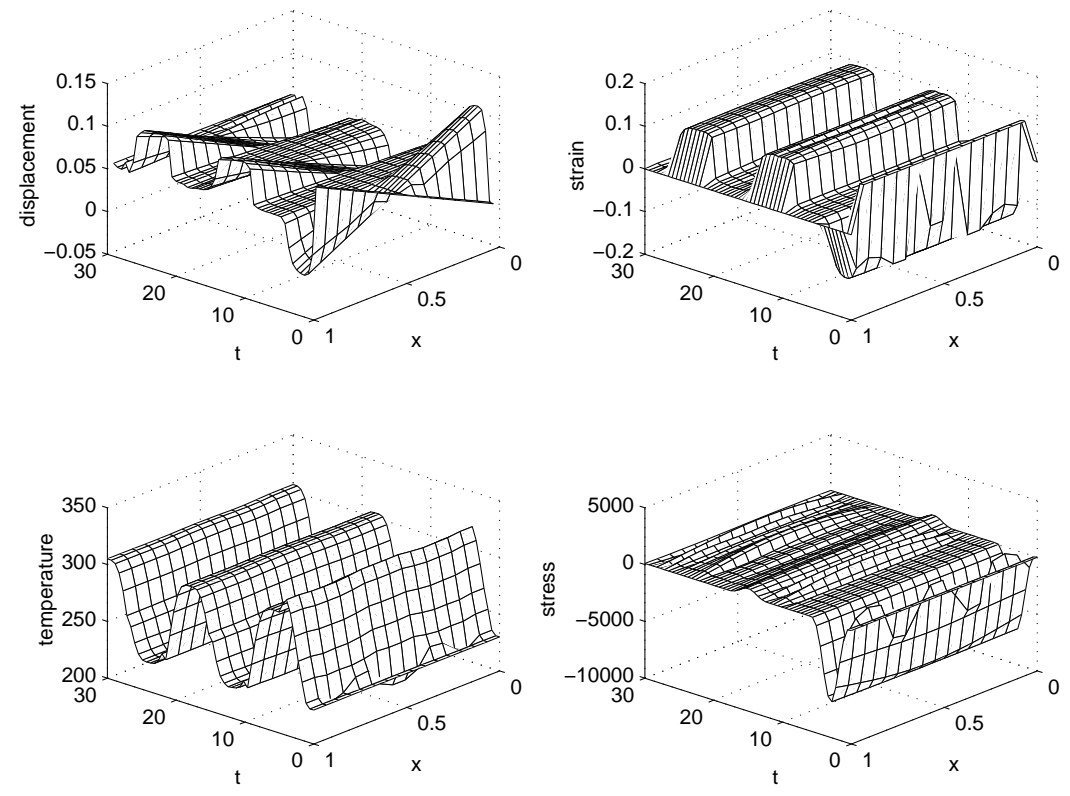

Figure 2. Temperature controls thermodynamic barriers allowing phase transitions even if the tensile (boundary) load is less than the yield limit.

Acknowledgments Fruitful collaboration on the topics of this paper with A. J. Roberts and K.A. Thomas of the University of Southern Queensland, Australia are gratefully acknowledged.

\section{REFERENCES}

[1] M. Auchenbach and I. Muller. Simulation of material behaviour of alloys with shape memory. Archives of Mechanics, 35 1985, 537-585.

[2] A. Bekker and L.C. Brinson. Temperature-induced phase transformation in a shape memory alloy: phase diagram based kinetics approach. J. Mech.Phys.Solids, 45 (6), 1997, 949-988. 
[3] A.D. Bruno. Normal forms. Mathematics and Computer in Simulation, 45 1998, 413427.

[4] J. Carr. Applications of Center Manifold Theory. Springer-Verlag, N.Y., 1981.

[5] S.-N. Chow, W. Liu, and Y. Yi. Center manifold for invariant sets. J. of Differential Equations, 168 2000, 355-385.

[6] J.M. Golden. Consequences of non-uniqueness in the free energy of materials with memory. Int. J. of Eng. Science, 39 2001, 53-70.

[7] S. Govindjee and G.J. Hall. A computational model for shape memory alloys. Int. J. of Solids and Structures, 37 2000, 735-760.

[8] K.-H. Hoffmann and J. Zou. Finite element approximations of Landau-Ginzburg's equation model for structural phase transitions in shape memory alloys. $M^{2} A N, \mathbf{2 9} 1995$, 629-655.

[9] L. Hsu, L.J. Min, and L. Favretto. A recursive approach to compute normal forms. Journal of Sound and Vibration, 243 (5), 2001, 909-927.

[10] P. Krejci, J. Sprekels, and S.M. Zhang. Asymptotic behaviour for a phase-field system with hysteresis. J. of Differential Equations, 175 2001, 88-107.

[11] D.C. Lagoudas. Modeling of the thermomechanical response of active laminates with SMA strips using the layerwise finite element method. J. of Intell. Mater. Syst. and Struct., 8 1997, 476-488.

[12] R. Mane. Persistent manifolds are normally hyperbolic. Trans. Amer. Math. Soc., 246 1977, 261-283.

[13] R.V.N. Melnik, A.J. Roberts, and K.A. Thomas. Modelling dynamics of shape-memoryalloys via computer algebra. In: Proceedings of SPIE, 3667, 1999, 290-301.

[14] R.V.N. Melnik, A.J. Roberts, and K.A. Thomas. Computing dynamics of copper-based SMA via center manifold reduction of 3D models. Computational Materials Science, $182000,255-268$.

[15] M. Niezgodka and J. Sprekels. Convergent numerical approximations of the thermomechanical phase transitions in SMA. Numer. Math., 58 1991, 759-778.

[16] E. Patoor et al.. Stress-induced martensitic transformation in polycrystalline alloys. ZAMM, Suppl. 2, 80 2000, 443-444.

[17] V.A. Pliss and G.R. Sell. Approximation dynamics and the stability of invariant sets. J. of Differential Equations, 149 1998, 1-51.

[18] V.A. Pliss and G.R. Sell. Perturbations of normally hyperbolic manifolds with applications to the Navier-Stokes equations. J. of Differential Equations, 169 2001, 396-492.

[19] A.J. Roberts. Low-dimensional modelling of dynamics via computer algebra. Computer Physics Communications, 100 1997, 215-230.

[20] A.J. Roberts. Computer algebra derives initial conditions for low-dimensional dynamical models. Comp. Phys. Commun., 126 2000, 187-206.

[21] J. Sprekels. Shape memory alloys: mathematical models for a class of first order solidsolid phase transitions in metals. Control and Cybernetics, 19 1990, 287-308.

\section{Fazinio pernešimo ir histerinès analizès terpèse su formos atmintimi taikymai}

\section{R.V.N. Melnik}

Straipsnyje centrinių daugdarų aproksimacija vienmačiams Landau-Devonskine ir trimačiams Falk-Konopka modeliams pritaikyta faziniams pernešimams terpèse su formos atmintimi. Metodologija leidžia sistemingai tikslinti foninio pernešimo reiškinio modelius. Pateikti tipiniai modeliavimo rezultatai mažos dimensijos modeliams 